\title{
A clinical study of annular cyclitis
}

\author{
Marilita Michael Moschos' \\ Yan Guex-Crosier ${ }^{2}$ \\ loannis Margetis' \\ Leonidas Zografos ${ }^{2}$ \\ 'Department of Ophthalmology, \\ University of Athens, Greece; ' Jules \\ Gonin Eye Hospital, University \\ of Lausanne, Switzerland
}

\begin{abstract}
Purpose: To investigate six cases of annular cyclitis.
Methods: All patients with impairment of visual acuity underwent complete ophthalmologic examination, color fundus photography, laboratory tests and fluorescein angiography. Indocyanine green (ICG) angiography and B-scan ultrasonography were also performed in three cases in order to diagnose the disease.

Results: All patients presented a unilateral or bilateral granulomatous uveitis, associated with inflammatory annular cyclitis. They had a shallow anterior chamber, a mildly elevated intraocular pressure (under $25 \mathrm{~mm} \mathrm{Hg}$ ) and an annular serous retinal detachment. A resolution was observed after specific therapy associated with systemic prednisolone therapy and antiglaucomatous drops.

Conclusion: This is the first description of an observational study of six patients with inflammatory annular cyclitis.
\end{abstract}

Keywords: cyclitis, uveitis, malignant glaucoma

\section{Introduction}

Annular cyclitis is a rare, chronic, usually bilateral uveitis, characterized by an inflammation of the ciliary body along with a low-grade anterior chamber inflammation and a peripheral retinal detachment, which may last for several years. It can be associated with secondary malignant glaucoma caused by the shallowing of the anterior (axial) chamber, its peripheral flattening and the increased intraocular pressure (IOP). However, very little is known about the pathology of annular cyclitis.

In our retrospective observational study, six cases of inflammatory cases of inflammatory annular cyclitis are described.

\section{Materials and methods}

Six patients were referred to the Jules Gonin Eye Hospital for evaluation of impairment of visual acuity (VA). All of them underwent ophthalmologic examination, which included measurement of VA on a standard Snellen chart, tonometry, binocular ophthalmoscopy, slit lamp biomicroscopy with a contact lens, color fundus photography, fluorescein angiography and laboratory tests. Indocyanine green (ICG) angiography and B-scan ultrasonography were also performed in three cases in order to diagnose the disease. Patients were regularly examined at 1-6-month intervals.

Characteristics and clinical findings for each patient are summarized in Table 1.

\section{Results}

Eight eyes of six patients were diagnosed with inflammatory annular cyclitis. Three $(50 \%)$ were male and three were female. Their ages ranged from 37 to 64 years (mean 52.11 years). The follow-up period ranged from three to six months (mean 5.4 months). Systemic medical and laboratory work-up was performed in order to diagnose the cause of cyclitis. Chest computed tomography established the diagnosis of systemic sarcoidosis in two patients. Mantoux test was positive (skin lesion $>20 \mathrm{~mm}$ ) in one patient. 
Table I Clinical findings and characteristics in patients with inflammatory annular cyclitis

\begin{tabular}{|c|c|c|c|c|c|c|}
\hline Patient no & I & 2 & 3 & 4 & 5 & 6 \\
\hline Diagnosis & TBC & Inflammatory scleritis & VKH & Sarcoidosis & Sarcoidosis & Idiopathic \\
\hline Age (years) & 61 & 61 & 37 & 48 & 41 & 64 \\
\hline Sex & M & M & $F$ & $\mathrm{~F}$ & $\mathrm{~F}$ & M \\
\hline Follow-up (months) & 6 & 3 & 6 & 6 & 4 & 5 \\
\hline Affected eye & OS & OD & OD & OD/OS & OD/OS & OD \\
\hline $\begin{array}{l}\text { Best-correctedVA } \\
\text { (initial) (OD/OS) }\end{array}$ & $1.0 / 0.5$ & $1.0 / 0.6$ & $1.0 / 0.6$ & $0.5 / 0.6$ & $0.6 / 0.7$ & $0.4 / 1.0$ \\
\hline $\begin{array}{l}\text { Best-corrected VA } \\
\text { (final) (OD/OS) }\end{array}$ & $1.0 / 0.5$ & $1.0 / 0.7$ & $1.0 / 0.8$ & $0.6 / 0.7$ & $0.7 / 0.7$ & $0.7 / 1.0$ \\
\hline Highest IOP (mm Hg) & 23 & 19 & 20 & $17 / 19$ & $21 / 18$ & 21 \\
\hline Treatment & $\begin{array}{l}\text { Anti-TBC drugs } \\
\text { and steroids }\end{array}$ & Steroids orally & Steroids orally & Steroids orally & Steroids orally & Steroids orally \\
\hline
\end{tabular}

Abbreviations: VKH, Vogt-Koyanagi-Harada; IOP, intraocular pressure; TBC, tuberculosis; CT, computed tomography.

All patients received systemic corticosteroids $(1 \mathrm{mg} / \mathrm{kg} / \mathrm{d})$ progressively tapered. We describe more extensively one illustrative case.

\section{Case I}

Patient 1, a 61-year-old man, had noticed impairment of visual acuity of OS for two months. The best-corrected visual acuity measured with a standard Snellen chart was found equal to $0.5 \mathrm{OS}$ and $1.0 \mathrm{OD}$. The examination OS revealed a relatively shallow, inflammatory anterior chamber with a ++ cellular activity and a ++ anterior chamber flare (Figure 1). The fundus examination OS showed multiple disseminated lesions of the posterior pole, associated with a mild cystoid macular edema (Figure 2). Indirect ophthalmoscopy revealed the presence of an annular cyclitis $\left(360^{\circ}\right)$ and a peripheral inferior serous retinal detachment. The examination OD revealed a relatively shallow, anterior chamber with a trace of cellular activity and a + anterior chamber flare. The fundus examination OD showed a temporally located inferior chorioretinal lesion. Systemic medical and laboratory work-up was performed in order to diagnose the cause of cyclitis. Mantoux test was positive (skin lesion $>20 \mathrm{~mm}$ ) in the patient and the diagnosis of systemic tuberculosis was established. The patient received anti-tuberculosis drugs without systemic steroids initially which were added after the first 10 days.

\section{Discussion}

Annular cyclitis is a rare disease, characterized by an inflammation of the ciliary body and, in some cases, associated with annular chorioretinal detachment. ${ }^{1}$

This is the first description, to our knowledge, of an observational case series of six patients with inflammatory annular cyclitis. It can be complicated with malignant-like

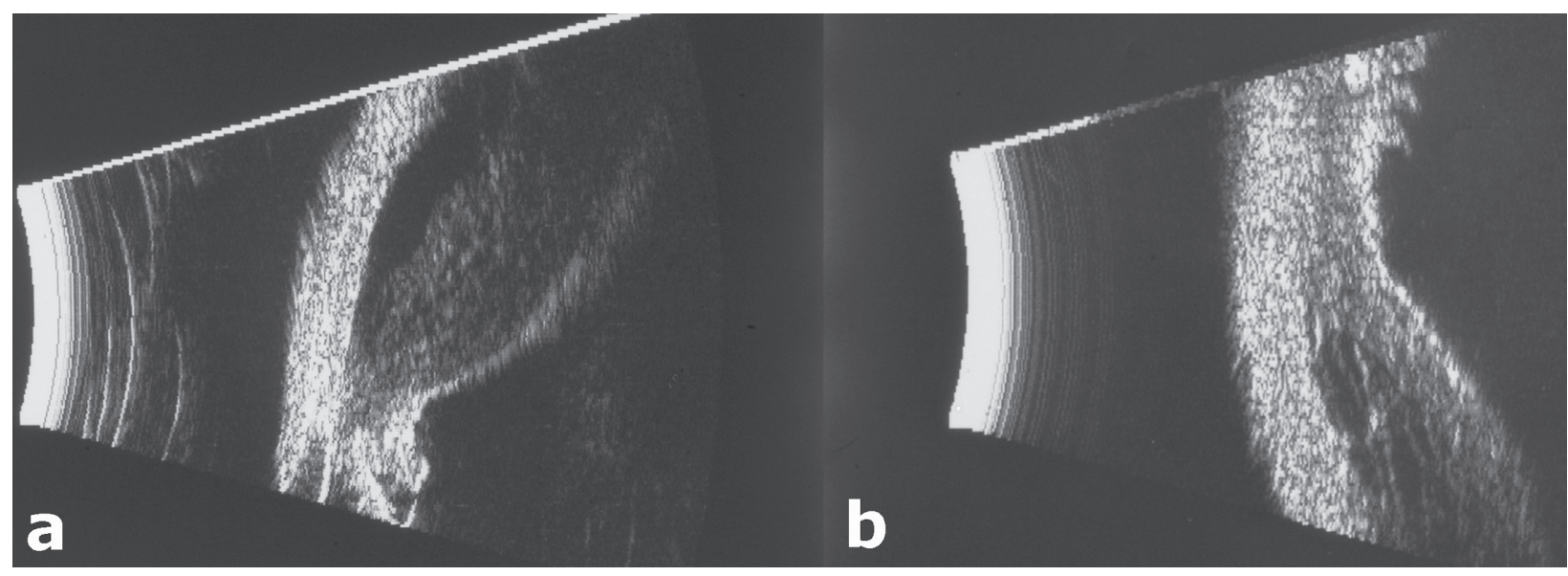

Figure I M-scan (Scheimpflug) photography showing shallowing of anterior chamber, angle-closure, and annular cyclitis OS A) compared to OD B). 


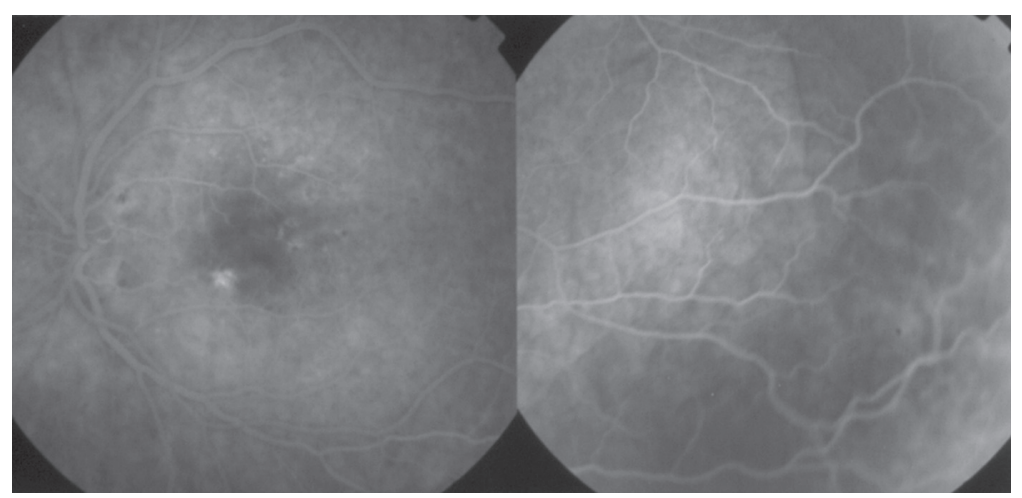

Figure 2 Late phase fluorescein angiogram OS showing multiple disseminated lesions of the posterior pole, mild cystoid macular edema, and serous retinal detachment.

glaucoma mainly caused by the shallowing of the anterior chamber due to the annular chorioretinal detachment. The term malignant-like glaucoma has been proposed for cases with similar clinical presentations with the malignant glaucoma but different underlying pathophysiology and causes. In this case series all patients present a potential risk of presenting a malignant-like glaucoma. This can be caused by the shallowing of the central anterior chamber, its peripheral flattening and the increased IOP. Also all patients were characterized by an absence of papillary block, an absence of suprachoroidal effusion or hemorrhage.

The differential diagnosis of annular cyclitis includes tumors of the ciliary body (ring melanomas, cysts), infectious diseases (Lyme, toxocariasis, syphilis, tuberculosis, fungal keratomycosis, Nocardia asteroides), systemic inflammatory diseases (sarcoidosis, Vogt-Koyanagi-Harada syndrome, rheumatic disease, inflammatory bowel disease).

Ring melanomas can closely simulate annular cyclitis ophthalmoscopically. In A-scan sonography the malignant melanoma of the ciliary body is characterized by low to medium reflectivity, whereas annular cyclitis combined with chorioretinal detachment manifests no internal reflectivity. ${ }^{2}$ After having excluded tumoral of infectious diseases, inflammatory agents should be suspected. Therefore, high dose of steroids should be introduced in order to be treated properly and rapidly. ${ }^{3}$

\section{Disclosure}

The authors report no conflicts of interest in this work.

\section{References}

1. Paufique L, Ravault MP, Bonnet M, et al. Annular chorio-retinal detachment or exudative cyclitis. Reflections apropos of a further case. Bull Soc Ophthalmol Fr. 1965;65(5):532-535.

2. Scherer U, Osterheld B. Echographic findings in cyclitis annularis pseudotumorosa and ring melanoma of the ciliary body. Klin Monatsbl Augenheilkd. 1985;187(5):455-456.

3. Kuo YH, Juang CJ. Sympathetic ophthalmia associated with cyclitis: case report. Changgeng Yi Xue Za Zhi. 1999;22(2):328-333. 
